\title{
The Information Cost Estimation as Realization of the Problem of Indistinct Mathematical Programming
}

 \\ Olegovna $^{4}$ \\ ${ }^{1}$ Doctor of Science, Economics, Russian Federation \\ ${ }^{2}$ Cand. Sc., Physics and Mathematics, Russian Federation \\ ${ }^{3}$ Ufa State Aviation Technical University, Russian Federation \\ ${ }^{4}$ Cand. Sc., St.Petersburg State Polytechnical University, Russian Federation \\ Correspondence: Lyudmila Nikolaevna Rodionova, Doctor of Science, Economics. E-mail: rodion@ufanet.ru
}

Received: January 9, 2015

Accepted: January 29, 2015

Online Published: July 15, 2015

doi:10.5539/mas.v9n8p186

URL: http://dx.doi.org/10.5539/mas.v9n3p186

\begin{abstract}
All information in the market has the consumer value. It is possible to estimate the recommended cost of investments in chance of reception of exact future result on the basis of calculation of consumer cost of the full information, and it is defined as a difference between expected values of a choice at presence or absence of the full information.

The problem of definition of cost of the information a priori is connected with necessity of the account of variety of the uncertain factors characterizing, firstly, its reliability, and, secondly its utility. It is possible to estimate reliability or utility of any information on the basis of the available statistical data, or expertly. Certainly, statistical methods allow to receive plausible enough estimations, however, by no means always the researcher possesses necessary statistical base that speaks or absence of the admission to it for privacy reasons, or exclusiveness of the information. The formalization of a problem of definition of information costing as a problem of indistinct mathematical programming, proceeding from purposes of the person, making decision (PMD), and on the basis of processing of the expert data is carried out in given work
\end{abstract}

Keywords: cost information, fuzzy mathematical programming

\section{Introduction}

The swift growth of rates of scientific and technical revolution and computerization of all fields of activity of a society has led to allocation of some concepts, seeming ordinary several years ago. The information and derived concepts generated by it become such phenomena occupying more and more significant place in our everyday life. The gain of information quantity circulating today in the world, has transformed it from a minor resource into the factor, fatefully influencing practically on all spheres of a public life, reflecting thereby increasing information dependence of a society.

Uncertainty is the characteristic peculiar to the majority of corporate administrative decisions. Usually deal with uncertainty of time and resources in realization of this or that purpose. One of the most difficult case for the analysis is the uncertainty connected with efficiency and costs at development of stocks or working out of a new kind of production when new technologies are introduced in manufacture.

In this work the problem is not to present the exhaustive list of all kinds of the uncertainty accompanying administrative decisions. The single aim is to underline that fact that the key problem lying before managers of the company is not to operate with uncertainty, but to explain it accurately at all stages of formation of decisions.

In the management theory it is supposed that the relevant information or, at least, the accessible information is given to the manager before he starts decision-making. But such situation appears extremely seldom in practice. One of the most important components of decision-making process is knowledge of what questions should be set. For example, the doctor cannot count that results of all possible trouble-shooting tests and interrogations will be given him by then as the patient will enter for the first time into his office.

The financier makes decisions when results are not defined and based on the limited information. There is often 
a choice of acceptance of strategies before him that is- the minimum profit at the minimum risks or the maximum profit at the maximum risks. For risk decrease the presence of the additional information which costs money is necessary for the inancier as a result of decision-making.

The concept of cost of the information is not defined in any Russian law. Consequently its calculation is not defined also. Cost of the state information is defined by assignment of a security label for the information of different level. It is possible to guess its cost indirectly on privacy degree. The private person or a business firm should define cost of that information which they would like to get or sell. In large firms there are security services, own or involved which estimate information cost. They are effective or not, it depends on their experience. Thus, as a rule, design procedure of cost of the information is not disclosed. All it allows to estimate efficiency of purchase and sale of the information rather conditionally.

The problem of definition of cost of the information a priori is connected with necessity of the account of variety of the uncertain factors characterizing, firstly, its reliability, and, secondly its utility. It is possible to estimate reliability or utility of any information on the basis of the available statistical data, or expertly. Certainly, statistical methods allow to receive plausible enough estimations, however, by no means always the researcher possesses necessary statistical base that speaks or absence of the admission to it for privacy reasons, or exclusiveness of the information. In such situation expert estimations remain hardly probable not the unique way, allowing to define information cost. Thus it is necessary to have in view that any expert estimations, being subjective reflexion of preferences, comprise uncertainty which is necessary to PMD for taking into consideration.

\section{Methods}

Let's available $m$ various types of information $I_{1}, \ldots, I_{m}$. Required cost of each type of information we will designate $x_{1}, \ldots, x_{m}$ accordingly. For definiteness we will believe that the target information is estimated only. We will input following assumptions: we will consider that each information possesses

utility for PMD $\alpha_{i}\left(x_{i}\right)$ which depends on cost of the information, (the given assumption is quite logical, considering that fact that if for the most valuable information unreasonably high price will be appointed, utility of such information will repeatedly decrease);

certain subjective reliability $\beta_{i}$ (in understanding PMD) which depends on degree of confidence PMD in utility of the information

Table 1. The Designations used for formalization of a problem of definition of cost of the information

\begin{tabular}{llll}
\hline Type of information & Information cost & Subjective reliability of the information & Utility of the information \\
\hline$I_{1}$ & $x_{1}$ & $\beta_{1}$ & $\alpha_{1}\left(x_{1}\right)$ \\
$I_{2}$ & $x_{2}$ & $\beta_{2}$ & $\alpha_{2}\left(x_{2}\right)$ \\
$I_{m}$ & $x_{m}$ & $\beta_{m}$ & $\alpha_{m}\left(x_{m}\right)$ \\
\hline
\end{tabular}

To set dependence of utility of the information $i$ of the kind on its cost it is equivalent to definition of function of utility. Certainly, it is the independent problem, which methods of the decision are well enough studied. Concerning functions of utility $\alpha_{i}\left(x_{i}\right), i=\overline{1, m}$ we will assume the following:

- All functions of utility $\alpha_{i}\left(x_{i}\right), i=\overline{1, m}$ change in a range from 0 to $1(1-$ the maximum utility, $0-$ minimum);

- Each function $\alpha_{i}\left(x_{i}\right)$ accepts the maximum value in some vicinity of point $x_{i}^{*}$ (or only in point $x_{i}^{*}$ ), and in process of removal from it utility falls to the minimum mark (the given assumption corresponds to a situation when PMD on the basis of the information available for it defines "fair" in its understanding the price of the information $i$ of th kind to deviate from which in the big party it is unprofitable for financial reasons, and in the smaller party - it is dangerous, in view of increase of risk of reception of the unfair information from "the unsatisfied" seller or possibility to lose the seller at all). 
Let's notice that the more PMD it is assured of "the fair" price defined by it, the there should be a reduction of values of function of utility $\alpha_{i}\left(x_{i}\right)$ in process of removal from point $x_{i}^{*}$ and on the contrary, than there is more than doubt PMD concerning "the fair" price faster, function $\alpha_{i}\left(x_{i}\right)$ in a vicinity of point $x_{i}^{*}$ should be especially flat.

Possible variants of graphic interpretations of functions of utility $\alpha_{i}\left(x_{i}\right)$ are more low resulted.



a) trapezoid function of utility



(b) triangular function of utility

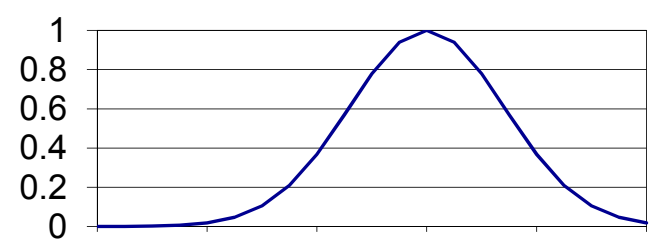

Gauss utility of function.

Figure 1. Graphic interpretations of functions of utility

In our opinion, the greatest interest is represented by Gauss functions of the utility which analytical task looks like

$$
\alpha(x)=e^{-\frac{(x-a)^{2}}{2 \sigma^{2}}}
$$

It is caused by that parameters $a$ and $\sigma$ such functions suppose simple enough interpretation: parameter $a$ represents the most probable value (population mean) of cost of information $x^{*}$, and parameter $\sigma$ - expected disorder of values of cost of the information from its most probable value $x^{*}$ (mean-square deviation). Both of these parameters can be defined on the basis of processing of the available statistical data, and on the basis of expert opinions. We will notice that on the basis of considered in a course of mathematical statistics of factor of a variation, on size $V=\sigma / a$ it is possible to judge Gauss "steepness" of a curve (than more factor of a variation, especially flat it is). It is obvious that the similar statement is fair and with reference to function of utility of a kind (1) (Figure 2 see). 


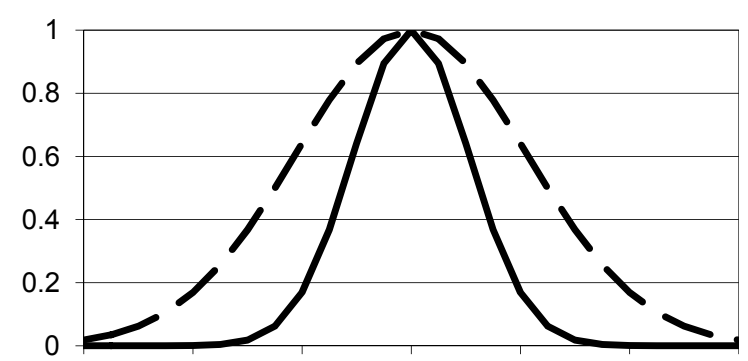

Figure 2. A type of Gauss function of utility at various values of factors of a variation

Varying parameters $a$ and $\sigma$, PMD can correct formalization of the representation about "the fair" price. And these representations should be considered at calculation of an estimation of reliability of the information: than more PMD it is assured of "the fair" price defined by it (i.e. the less size $V=\sigma / a$ ), the there should be in understanding PMD a reliability of information $\beta$, and on the contrary more. Thus, size $V=\sigma / a$ can be taken as a principle definitions of subjective reliability of the information. In the present work $\beta$ it is offered to count as follows:

$$
\beta(V)=e^{-\left(\frac{\sigma}{a}\right)^{2}}
$$

Graphic representation of function $\beta(V)$ is represented on Figure 3.



Figure 3. Graphic interpretation of function of reliability $\beta(V)$

Thus, having formalized the function of utility (having set parameters $a$ and $\sigma$ ), PMD can automatically estimate and subjective reliability of the information.

Lets name the size $\beta_{i} \alpha_{i}\left(x_{i}\right)$ the corrected utility $i$ of the information. Then size

$$
\sum_{i=1}^{m} \beta_{i} \alpha_{i}\left(x_{i}\right)
$$

The size will define the total corrected utility of all set of the got information, and

$$
\frac{1}{m} \sum_{i=1}^{m} \beta_{i} \alpha_{i}\left(x_{i}\right)
$$


The average total corrected utility of the got information. We will notice that in the total corrected utility of the information (3) unlike simple total utility $\sum_{i=1}^{m} \alpha_{i}\left(x_{i}\right)$ the factors of uncertainty inherent in process of definition PMD of the "fair" prices are considered.

\section{Results}

Using the entered sizes and the approach described above, it is possible to formulate or a problem of definition of cost of the got information minimizing total expenses, under condition of achievement of the set level of the average total corrected utility of the got information, or a problem of definition of cost of the got information, maximizing average total corrected utility of the got information, in the conditions of limitation of means for information acquisition.

In the first case the model will look like:

$$
\begin{gathered}
\sum_{i=1}^{m} x_{i} \rightarrow \min \\
\frac{1}{m} \sum_{i=1}^{m} \beta_{i} \alpha_{i}\left(x_{i}\right) \geq \gamma \\
x_{i} \geq 0, \quad i=\overline{1, m},
\end{gathered}
$$

Where $\gamma$ is the set level of the average total corrected utility of the got information. We will notice that size $\frac{1}{m} \sum_{i=1}^{m} \beta_{i} \alpha_{i}\left(x_{i}\right)$ accepts values in a range from 0 (the worst value) to 1 (the best value). Therefore level $\gamma$ has simple enough and evident interpretation: $\gamma$ corresponds to the minimum comprehensible share from the best value of the average total corrected utility of the got information.

For the second of the formulated problems the model will register in a kind:

$$
\begin{gathered}
\frac{1}{m} \sum_{i=1}^{m} \beta_{i} \alpha_{i}\left(x_{i}\right) \rightarrow \max \\
\sum_{i=1}^{m} x_{i} \leq S \\
x_{i} \geq 0, \quad i=\overline{1, m}
\end{gathered}
$$

Where $S$ is the size characterizing the top border of the sum which PMD assumes to spend for information acquisition.

\section{Discussion}

Let's stop on a problem of an estimation of uncertainty of the available information. The availability of the factor of uncertainty is caused by that PMD can not possess 100 percent confidence at definition of functions of utility of the information that is in turn expressed in the task of parameters $\alpha$ and $\sigma$ in a random way. Further it leads to uncertainty, both at an estimation of subjective reliability of the information, and at definition of its cost. Certainly, uncertainty degree varies for various situations.For practice it is important to have possibility to make a numerical estimation of uncertainty in each situation. As such measure we will use entropy which is equal to average uncertainty of all possible outcomes:

$$
H(x)=-\sum_{i=1}^{n} p_{i} \log _{2} p_{i}
$$

Where $x$ is one of independent events with $n$ possible conditions $\mathrm{Pi}$ - probability $i$ of the condition. Thus size $\log _{2} \frac{1}{p_{i}}$ is called as private entropy and characterizes only $i$ condition. For practice entropy is important that allows comparing uncertainty of various experiences to casual outcomes. 
In our case event $x$ results from definition PMD of parameters of all functions of utility $\alpha_{i}\left(x_{i}\right)$. Each of such events we will consider independent from similar others. The given assumption is fair, if PMD makes decisions, being based only on the knowledge, instead of on results of the last ways of definition of functions of utility.

Event $x$ leads to definition of sizes of subjective reliability of information $\beta_{i}, i=\overline{1, m}$. Owing to a way of their calculation the given sizes can be interpreted as probability of approach of corresponding events $A_{i}=\ll i$ th information is authentic in understanding PMD», and sizes $\left(1-\beta_{i}\right)$ - as probabilities of approach of opposite events $\overline{A_{i}}=\ll i$ th information is not authentic in understanding PMD». Thus, following $2^{m}$ of variants of combinations of events is possible:

$$
\begin{aligned}
& A_{1}, \overline{A_{2}}, \overline{A_{3}}, \ldots, \overline{A_{m}}, \\
& \overline{A_{1}}, A_{2}, \bar{A}_{3}, \ldots, \overline{A_{m}}, \\
& A_{1}, A_{2}, \overline{A_{3}}, \ldots, \overline{A_{m}}, \\
& \overline{A_{1}}, \overline{A_{2}}, \overline{A_{3}}, \ldots, \overline{A_{m}},
\end{aligned}
$$

Which probabilities are accordingly equal to

$$
\begin{gathered}
\beta_{1}\left(1-\beta_{2}\right)\left(1-\beta_{3}\right) \ldots\left(1-\beta_{m}\right) \\
\left(1-\beta_{1}\right) \beta_{2}\left(1-\beta_{3}\right) \ldots\left(1-\beta_{m}\right) \\
\ldots \ldots \\
\beta_{1} \beta_{2}\left(1-\beta_{3}\right) \ldots\left(1-\beta_{m}\right) \\
\ldots \ldots \\
\left(1-\beta_{1}\right)\left(1-\beta_{2}\right)\left(1-\beta_{3}\right) \ldots\left(1-\beta_{m}\right) .
\end{gathered}
$$

Events (12) form full group, therefore the entropy generated by event $x$, will pay off under the formula:

$$
H(x)=-\sum_{\substack{i_{1}=\overline{0,1} \\ \ldots \ldots \ldots .}}\left(1-\beta_{1}\right)^{1-i_{1}} \beta_{1}^{i_{1}} \ldots\left(1-\beta_{m}\right)^{1-i_{m}} \beta_{m}^{i_{m}} \log _{2}\left[\left(1-\beta_{1}\right)^{1-i_{1}} \beta_{1}^{i_{1}} \ldots\left(1-\beta_{m}\right)^{1-i_{m}} \beta_{m}^{i_{m}}\right] .
$$

It is known that entropy is maximum, if all outcomes are equiprobable. In case of described above the approach to entropy calculation its maximum value is equal

$$
H_{\max }=-\sum_{i=1}^{2^{m}} \frac{1}{2^{m}} \log _{2} \frac{1}{2^{m}}=2^{m} \frac{1}{2^{m}} \log _{2} 2^{m}=m
$$

On the basis of the received value of expression (14) it is possible to judge degree of uncertainty of the available information formalized according to representations PMD. Thus it is possible to compare various sets of the information among themselves (more uncertain will be considered at what value of entropy above) or to compare 
entropy of set of information $H(x)$ to the maximum value $m$. Also during research by the useful there can be an analysis of values of private entropies.

$$
\begin{gathered}
\log _{2} \frac{1}{\left(1-\beta_{1}\right)^{1-i_{1}} \beta_{1}^{i_{1}} \ldots\left(1-\beta_{m}\right)^{1-i_{m}} \beta_{m}^{i_{m}}}, \\
i_{1}=\overline{0,1}, \ldots, i_{m}=\overline{0,1} .
\end{gathered}
$$

To help PMD to understand the assumptions put forward by it and to facilitate a problem of definition of exact restrictions, transformation of problems of mathematical programming (5) - (7) and (8) - (10) in problems of indistinct mathematical programming is expedient. In problems of indistinct mathematical programming probably to consider situations when about some parameters of model that they are in some set reflecting real (or desirable) possibilities is known only, and also situations in which instead of maximization (minimization) of criterion function it is enough that it reached only some set level, thus to various deviations of criterion function from the given level various degrees of an admissibility can be attributed.

Let's admit that PMD, solving a problem (5) - (7), it is not assured of correctness of unequivocal definition subjective reliable $\beta_{i}, i=\overline{1, m}$, and considers that more correct will consider their range of possible values, and concerning the average total corrected utility of got information $\gamma$ can assert only that $\gamma \in\left[\gamma^{\min }, \gamma^{\max }\right]$. In this case it is possible to speak about the indistinct task of parameters $\beta_{i}, i=\overline{1, m}$ and $\gamma$, and, as consequence, about statement of a problem of indistinct mathematical programming (5) - (7).

Let's set a range of possible values for $\beta_{i}$ as follows:

$$
\beta_{i} \in\left[\beta_{i}^{\min } ; \beta_{i}^{\max }\right]
$$

Where $\beta_{i}^{\min }=\beta_{i}(1-\delta), \beta_{i}^{\max }=\min \left\{\beta_{i}(1+\delta) ; 1\right\}, \delta \in[0 ; 1]-$ is a constant, setting which, PMD can vary width of corridors for possible values $\beta_{i}, i=\overline{1, m}$. It is obvious that the more $\delta$, the there is less than confidence at PMD concerning correctness $\beta_{i}$.

Owing to definition of functions of utility $\alpha_{i}\left(x_{i}\right), i=\overline{1, m}$ a problem (5) - (7) with indistinct parameters $\beta_{i}$, $i=\overline{1, m}$ and $\gamma$ can lead the problem with accurately certain parameters as follows:

$$
\begin{gathered}
\sum_{i=1}^{m} x_{i} \rightarrow \min \\
\frac{1}{m} \sum_{i=1}^{m} \beta_{i}^{\min } \alpha_{i}\left(x_{i}\right) \geq \gamma^{\min } \\
\frac{1}{m} \sum_{i=1}^{m} \beta_{i}^{\max } \alpha_{i}\left(x_{i}\right) \leq \gamma^{\max } \\
x_{i} \geq 0, \quad i=\overline{1, m} .
\end{gathered}
$$

As a result of the decision of a problem (18) - (21) at preset value $\delta$ the vector of optimum cost of information 
$X^{*}=\left\{x_{1}^{*} ; \ldots ; x_{m}^{*}\right\}$ and optimum value of criterion function $f^{*}=\sum_{i=1}^{m} x_{i}^{*}$ will be defined. Number $1-\delta$ can be considered as accessory and vector $X^{*}$ degree to indistinct set of decisions, and simultaneously, as degree of accessory $f^{*}$ to indistinct set of optimum values of criterion function of an initial problem. We will notice that

if $\delta=0$ the problem (18) - (21) is not indistinct, and, hence, decision $X^{*}$ is unequivocally optimum, and $f^{*}$

- minimum of all possible values of criterion function. Varying possible values $\delta$, it is possible to receive functions of an accessory of the indistinct decision and indistinct value of criterion function $[1,2,15,16]$.

Let's assume that, solving a problem (8) - (11), PMD do not aspire to maximize the average total corrected utility, and to aspire to that the given size has reached some comprehensible level $\gamma$, and let there is threshold level $\gamma$, less which criterion function (8) should not accept value. Then function of an accessory to indistinct function of the purpose can be defined as follows:

$$
\mu_{f_{0}}(X)=\left\{\begin{array}{l}
0, \quad \text { if } \sum_{i=1}^{m} x_{i} \leq \gamma^{0}, \\
\mu_{\gamma}(X), \text { if } \gamma^{0} \leq \sum_{i=1}^{m} x_{i}<\gamma^{*}, \\
1, \quad \text { if } \sum_{i=1}^{m} x_{i} \geq \gamma^{*} .
\end{array}\right.
$$

Where $\mu_{\gamma}(X)$ - the function of an accessory describing degrees of performance of the corresponding inequality from the point of view of PMD.

\section{Conclusion}

Function of accessory $\mu_{S}(X)$ for indistinct restriction (9) can be similarly defined. Indistinct conditions can be considered as set of alternatives $X$ together with its indistinct subsets representing indistinctly formulated criteria (purpose $f_{0}$ and restriction $\left.f_{1}\right)$, i.e. as system $\left(X, f_{0}, f_{1}\right)$. As a result the initial problem (8) - (11) can be formulated in the form of a performance problem of indistinctly definite purpose to which we will apply the approach of Bellman - Zade according to which is to take into consideration whenever possible all criteria in such problem means to construct function into which the purposes and restrictions are included equally $[18,19,20]$ :

$$
D=f_{0} \cap f_{1}
$$

The decision can be defined as an indistinct subset of universal set of possible values. The optimum corresponds to that area which elements maximize $D$. Thus, and the problem (8) - (11) can be shown to a problem of indistinct mathematical programming.

\section{References}

Altunin, A. E. (2010). Semukhin M.V Models and algorithms of decision making under uncertainty. Tyumen: TSU Publishing House, 352.

Berndt E. R. (2005). Econometrics practice. - M.: Yuniti-Dana.

Derevyanko, P. M. (2003). Elements of fuzzy logics in investment portfolio formation. Economics and infocomms in XXI century: Proceedings of the II International scientific and practical conference. November 24-29. SPb.: SPSUE Publishing House, 317-319.

Derevyanko, P. M. (2005). Comparison of indeterminate and simulation approach to modeling of enterprise activity under uncertainty. Modern problems of economics and management of national economy. Collection of scientific articles. Issue. SPb.: SPSUE, 289-292.

Derevyanko, P. M. (2005). Fuzzy modeling of enterprise activity and evaluation of risks of making strategic financial decision under uncertainty. Modern problems of applied informatics: I scientific and practical conference, May 23-25. Book of reports - SPb.: SPSUE, 81-83.

Doherty K. (1997). Introduction in econometrics. -M.: INFRA-M. XIV, 402 p.: ill. - (University book) Literature 
reference: $384-386$.

Dubois, D., \& Prade, H. (1990). Possibility Theory. Applications to presentation of knowledge on informatics. Translated from French into Russian. - M. Radio and communication. 288.

Eliseeva, I. I. (2001). Econometrics: Textbook/I.I. Eliseeva et al., M.: Finances and Statistics.

Kahraman, C., Ruan, D., \& Tolga, E. (2002). Capital Budgeting Techniques Using Discounted Fuzzy versus Probabilistic Cash Flows. Information Sciences, 142, 57-76.

Kleiner, G. B. (2001). Economical and mathematical modeling and economic theory. Economics and Mathematical Methods, 37(3).

Kleiner, G. B., Tambovtsev, V. L., \& Kachalov, R. M. (1997). Enterprise in unstable economical environment: risks, strategy, safety. M.: Economics.

Knyazevskiy, V. S., \& Zhitnikov, I. V. (2014). Analysis of time series and prediction: Learning guide. Rostov-on-Don: RSEA, 161.

Kofman, A., \& Hil, A. (2012). Introduction to the theory of fuzzy sets in the management of enterprises: Per. with App. - Mn .: Higher School, $224 \mathrm{p}$.

Saati T. L. (2010). Decision making. Hierarchy Analysis Method. - M.: Radio and communication, 316.

Saati, T. L. (2008). Decision making under dependences and feedback: Analytical networks. — M.: Publishing House LKI, 360.

Sobol, I. M., \& Statnikov, R. B. (1981). A choice of optimum parameters in problems with many criterias. M: The Science.

Tsarev, V. V. (2014). Evaluation of economical efficiency of investments.

Voschinin, A. P. (2014). Problems of analysis with ambiguous data - intervals and/or randomness? Interval mathematics and constraint propagation: Business meetings, 147-158.

Zade, L. (1974). The basis of the new approach to the analysis of difficult systems and decision-making processes. Mathematics today: transl. from English - M: Znanie, 5-48.

\section{Copyrights}

Copyright for this article is retained by the author(s), with first publication rights granted to the journal.

This is an open-access article distributed under the terms and conditions of the Creative Commons Attribution license (http://creativecommons.org/licenses/by/3.0/). 